\title{
RADIOTHYROXINE TURNOVER STUDIES IN THYROID DISEASE AFTER THERAPY
}

\author{
By KENNETH STERLING \\ (From the Department of Internal Medicine, New York State Psychiatric Institute, and the \\ Department of Psychiatry, Columbia University College of Physicians and \\ Surgeons, New York, N. Y.; and the Department of Medicine, State \\ University of New York, Upstate Medical Center, \\ Syracuse, N.Y.)
}

(Submitted for publication April 21, 1958; accepted June 19, 1958)

In a previous report the disappearance curve of plasma radioactivity after intravenous injection of I $^{131}$-labeled 1-thyroxine was employed to determine the rate of hormone degradation or removal in subjects with thyroid disease (1). Thyroxine degradation was slower than normal in untreated myxedema, and faster than normal in

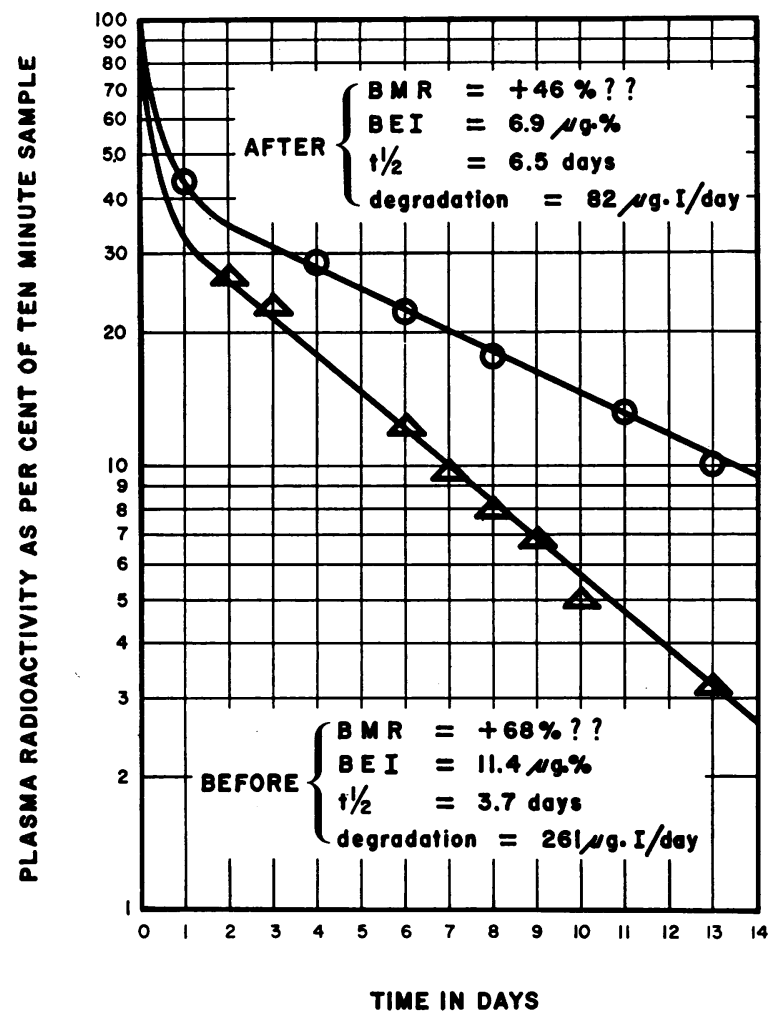

Fig. 1. Radiothyroxine Disappearance Curve in Thyrotoxicosis-SubJect L.S., No. 13, Studied Before ANd Eight Months After I ${ }^{131}$ Therapy

Semilogarithmic plot. Points after 14 days are not illustrated. Degradation rates are corrected to $1.73 \mathrm{M}^{2}$ surface area. The accuracy of basal metabolism values is uncertain. thyrotoxicosis. These findings were in agreement with those of Berson and Yalow (2) and Ingbar and Freinkel (3).

The present work was undertaken to observe the effects of treatment and attainment of the euthyroid state, with studies on individual subjects before and after therapy.

\section{MATERIAL AND METHODS}

$\mathrm{I}^{131}$-labeled 1-thyroxine was obtained in $1 \mathrm{mc}$. shipments from Abbott Laboratories, Oak Ridge, Tenn. The radiothyroxine was diluted in 50 to $75 \mathrm{ml}$. sterile saline to which approximately $50 \mathrm{mg}$. Red Cross albumin had been added to prevent adsorption by vessel walls. The injections were carried out within one to two hours of the preparation of dilute solutions. The subjects received tracer amounts (approximately $50 \mu \mathrm{c}$. in 2 to $10 \mu \mathrm{g}$. thyroxine) by intravenous injection. Heparinized venous blood samples were taken 10 minutes after injection and daily or on alternate days for two weeks or longer. The plasma radioactivity was assayed in a well-type scintillation counter which recorded approximately one million counts per minute $(\mathrm{cpm})$ per microcurie of $I^{181}$ (42 per cent overall efficiency) above a background of $130 \mathrm{cpm}$. Corrections for radioactive decay were made when necessary. Usually all plasma samples from a given patient and diluted aliquots of the administered compound were counted together after conclusion of the study, obviating the need for decay correction.

In all subjects two or more plasma protein bound iodine (PBI) or butanol extractable iodine (BEI) determinations were carried out $1(4,5)$. The BEI was done to avoid interference from previously administered inorganic iodine. The two determinations were used interchangeably in the calculations of "organic iodine" figures; PBI ordinarily exceeds BEI by approximately $0.6 \mu \mathrm{g}$. per cent in the absence of inorganic iodine (5).

Two or more basal metabolic rate (BMR) determinations were performed on separate days, usually during the period of study.

Iodine prefeeding was employed to prevent reutiliza-

1 Performed by Bio-Science Laboratories, Los Angeles, Calif. 


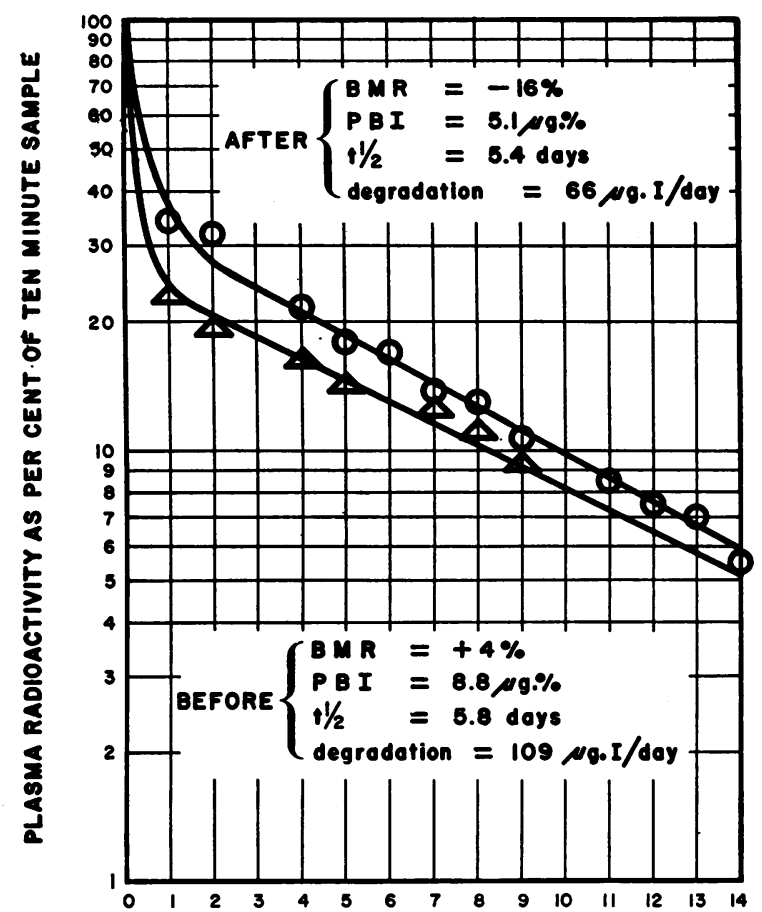

TIME IN DAYS

Fig. 2. Radiothyroxine Disappearance Curve in Thyrotoxicosis-Subject M.O., No. 12, Studied Before ANd 15 Months After ${ }^{181}$ Therapy

Semilogarithmic plot. Points after 14 days are not illustrated. Degradation rates are corrected to $1.73 \mathrm{M}^{2}$ surface area.

tion of the $\mathrm{I}^{131}$-label in the thyrotoxic subjects only. Both treated and untreated thyrotoxic subjects received Lugol's solution, 15 drops daily, prior to and throughout studies. The myxedematous and normal control subjects did not receive iodine prefeeding since previous studies $(1,3)$ indicated that the error would be negligible except in hyperthyroid subjects.

Calculations. The injection of radiothyroxine was followed by a relatively rapid fall in the radioactivity of the plasma (Figures 1 through 4 ). This was attributed to the diffusion of the tracer out of the plasma throughout the body's extrathyroidal organic iodine (EOI) pool. After two days the plasma radioactivity declined more gradually, forming a straight line when plotted semilogarithmically (Figures 1 through 4 ). This slow exponential component of the disappearance curve was interpreted as metabolic degradation of the administered radiothyroxine, hence a measure of the turnover rate of the hormone. The computations employed have been described in detail previously (1). The halftime of thyroxine turnover was obtained graphically from the linear component of the disappearance curve. The turnover rate was computed from the half-time by the equation $k=\ln 2 / t^{1} / 2$, where $t^{1} / 2$ represents the half-time in days, and $k$, the turnover rate as the fraction of the body's extrathyroidal organic iodine (EOI) pool synthesized and degraded daily. The turnover rate, $k$, was conveniently expressed as per cent per day.

The EOI pool was estimated from the quotient:

\section{Total radioactivity injected $\widehat{\text { Radioactivity per microgram of PBI }}$}

The denominator was obtained from the zero time extrapolation which represented plasma radioactivity if distribution had occurred instantaneously after injection.

The product of the EOI pool and the turnover rate (k) gave the degradation rate in micrograms of organic iodine per day. The rates of degradation and formation should be the same in a steady state, which was assumed to exist in the absence of significant alterations in the clinical condition. The term "degradation rate" signifies rate of removal of thyroxine from the extrathyroidal pool without specification of precise metabolic pathway or route.

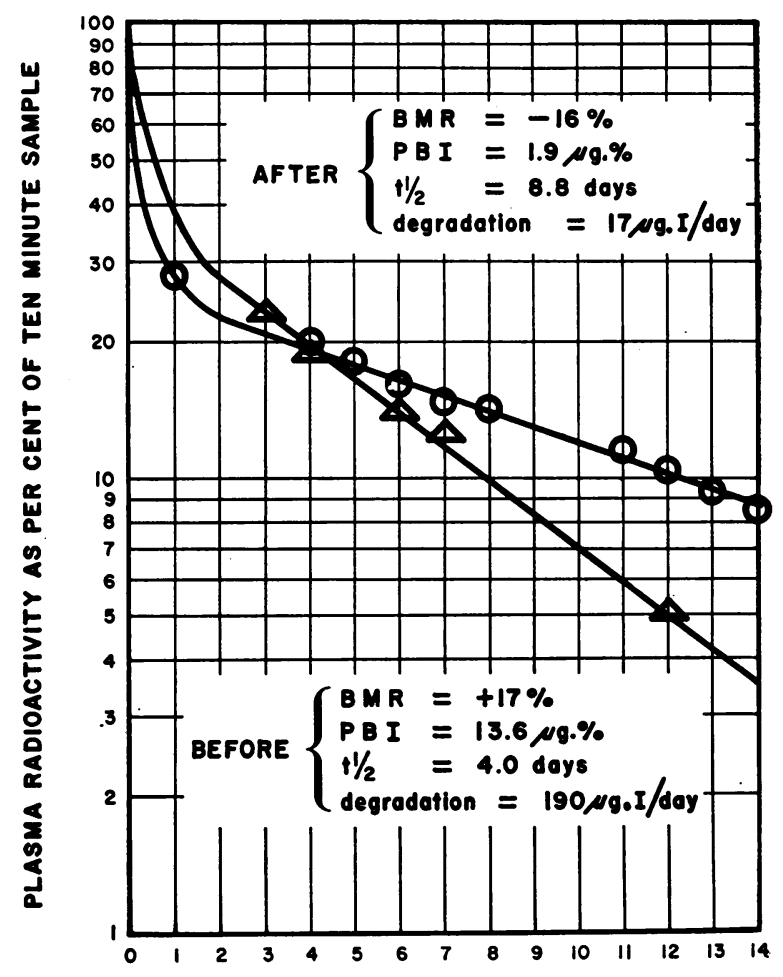

TIME IN DAYS

Fig. 3. Radiothyroxine Disappearance CURve in Thyrotoxicosis-SubJect R.D., No. 11, Studied Before ANd ONe Year After I ${ }^{191}$ Therapy

Semilogarithmic plot. Points after 14 days are not illustrated. Degradation rates are corrected to $1.73 \mathrm{M}^{2}$ surface area. 

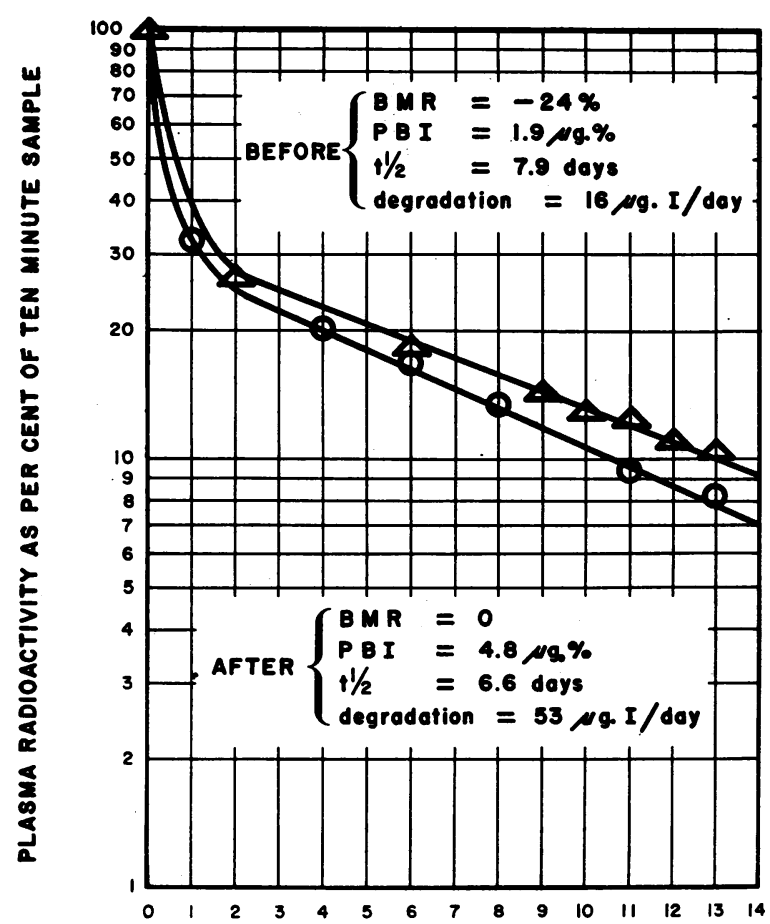

TIME IN DAYS

Fig. 4. Radiothyroxine Disappearance Curve in Myxedema-Subject H.L., No. 1, Studied Before and One Year After Replacement Therapy

Semilogarithmic plot. Points after 14 days are not illustrated. Degradation rates are corrected to $1.73 \mathrm{M}^{2}$ surface area.

Clinical material. The following groups were studied:

Normal. Ten healthy medical student volunteers served as control subjects.

Thyrotoxicosis. Twenty cases were studied after treatment and attainment of euthyroid status, or in certain specified instances, hypothyroid status, as judged by clinical appearance, PBI and BMR determinations. No patients with evident residual toxicity were chosen for study. Of this group six were also studied prior to treatment while still toxic, five having been reported previously (1). The treatment was subtotal thyroidectomy in three cases, and $\mathrm{I}^{131}$ in the rest, usually supplemented by antithyroid drug (propylthiouracil or Tapazole ${ }^{\circledR)}$ for two months beginning one week after $\mathrm{I}^{131}$. No patient received intithyroid drug during the studies except M.O., No. 12. All patients of the thyrotoxic group received Lugol's solution, 15 drops daily, prior to and throughout studies, with the exception of M.O., No. 12, who received antithyroid drugs during the initial study, as described subsequently.

The diagnosis was diffuse toxic goiter (Graves' discase) in all cases except the following :

nodular toxic goiter in Nos. 12, 22, 23, 25 and 27 of Table I; recurrent toxic goiter, status p.o. thyroidectomy, in Nos. 18, 20 and 26; and

struma lymphomatosa (Hashimoto) in No. 10, verified histologically.

Myxedema. Eight cases of myxedema were studied after various periods of replacement therapy with desiccated thyroid which, except in specified instances, had restored euthyroid status, as judged by clinical appearance, PBI and BMR determinations. All but one case had studies before therapy, five having been reported previously (1). Two of the eight cases (Nos. 2 and 7) had myxedema following resection of nontoxic goiter. The rest had spontaneous idiopathic myxedema.

\section{RESULTS}

The findings are listed in Table I, and illustrated in Figures 1 through 5. Definite changes were observed following treatment, and were especially pronounced in the thyrotoxic subjects. In subjects studied both before and after treatment, the changes in turnover rates are depicted in Figure 5, which shows changes in the same direction in all instances except one in each group.

\section{Thyrotoxicosis}

Figure 1 was selected to demonstrate the marked slowing of disappearance rate which was observed after treatment of thyrotoxicosis. The very short half-time had returned to the normal range. All treated thyrotoxic subjects except M.O., No. 12 (Figure 2), had slowing to a normal disappearance curve (Figure 1) or even slower than normal (Figure 3).

The exceptional case, M.O., No. 12 (Figure 2), showed no appreciable change in disappearance curve after $\mathrm{I}^{131}$ treatment and attainment of the euthyroid state. The disappearance rate was slightly accelerated in the initial and final studies. The patient had been maintained nearly euthyroid with antithyroid medications begun three weeks before and continued during the initial study (Tapazole ${ }^{\circledR}, 40 \mathrm{mg}$. daily; later propylthiouracil, $400 \mathrm{mg}$. daily). Although the disappearance curve remained the same, the pool size and degradation rate fell markedly after $\mathrm{I}^{131}$ treatment (Table I and Figure 2) in a study carried out during the administration of Lugol's solution.

In Subject L.S., No. 13, illustrated in Figure 1, despite the marked slowing of disappearance rate there was still some elevation of the pool size and degradation rate (Table I). 
TABLE I

Data from radiothyroxine disappearance curves including values adjusted to $1.73 \mathrm{M.2}$ surface area and $70 \mathrm{Kg}$. body weight

\begin{tabular}{|c|c|c|c|c|c|c|c|c|c|c|c|c|c|c|c|c|}
\hline \multirow[b]{3}{*}{$\begin{array}{l}\text { Condi- } \\
\text { tion }\end{array}$} & \multirow[b]{3}{*}{$\begin{array}{l}\text { Sub- } \\
\text { ject }\end{array}$} & \multirow[b]{3}{*}{ Sex } & \multirow[b]{3}{*}{$\begin{array}{c}\text { Age } \\
y r s .\end{array}$} & \multirow[b]{3}{*}{$\begin{array}{l}\text { Wt. } \\
\mathbf{K g} .\end{array}$} & \multirow[b]{3}{*}{$\begin{array}{l}\text { Sur- } \\
\text { face } \\
\text { area } \\
M .^{2} \\
\end{array}$} & \multirow[b]{3}{*}{ Therapy* } & \multirow[b]{3}{*}{ BMR† } & \multirow[b]{3}{*}{$\begin{array}{c}\text { PBI } \\
\mu g . \%\end{array}$} & \multicolumn{8}{|c|}{ Kinetic and isotope dilution data } \\
\hline & & & & & & & & & \multicolumn{2}{|c|}{$\begin{array}{c}\begin{array}{c}\text { Kinetics of } \\
\text { disappearance }\end{array} \\
\end{array}$} & \multicolumn{3}{|c|}{$\begin{array}{c}\text { Extrathyroidal organic } \\
\text { iodine pool } \\
\text { (EOI) }\end{array}$} & \multicolumn{3}{|c|}{ Degradation rate } \\
\hline & & & & & & & & & $\begin{array}{c}\text { Half- } \\
\text { time } \\
\text { ti } \\
\text { days }\end{array}$ & $\begin{array}{l}\text { Turn- } \\
\text { over } \\
\text { rate k } \\
\text { \%/day }\end{array}$ & $\mu \mathrm{g}$. & $\underset{\mathrm{Mg} . \mathrm{i}^{\mathrm{\mu g}} / 1.73}{ }$ & $\begin{array}{c}\boldsymbol{\mu g} . / 70 \\
\mathbf{K g} .\end{array}$ & $\mu g . /$ day & $\begin{array}{l}\mu \mathrm{gg} . / 1.73 \\
\mathrm{M} .2 / \mathrm{day}\end{array}$ & $\begin{array}{c}\mu \mathrm{g} . / 70 \\
\text { Kg./day }\end{array}$ \\
\hline \multirow{9}{*}{ 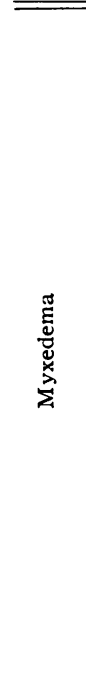 } & H. L. & $\mathbf{F}$ & $\begin{array}{l}40 \\
41\end{array}$ & $\begin{array}{l}103.6 \\
104.6\end{array}$ & $\begin{array}{l}2.12 \\
2.13\end{array}$ & $\begin{array}{c}0 \\
180 \mathrm{mg} . \\
13 \mathrm{mos} .\end{array}$ & -24 & $\begin{array}{l}1.9 \\
4.8\end{array}$ & $\begin{array}{l}7.9 \\
6.6\end{array}$ & $\begin{array}{r}8.8 \\
10.5\end{array}$ & $\begin{array}{l}233 \\
619\end{array}$ & $\begin{array}{l}191 \\
503\end{array}$ & $\begin{array}{l}158 \\
414\end{array}$ & $\begin{array}{l}20 \\
65\end{array}$ & $\begin{array}{l}16 \\
53\end{array}$ & $\begin{array}{l}14 \\
43\end{array}$ \\
\hline & $\underset{2}{M}$. A. & $\mathbf{F}$ & $\begin{array}{l}58 \\
59\end{array}$ & $\begin{array}{l}43.3 \\
39.1\end{array}$ & $\begin{array}{l}1.44 \\
1.37\end{array}$ & $\begin{array}{l}\text { 0 } \\
90 \mathrm{mg} . \\
6 \text { mos. }\end{array}$ & $\begin{array}{l}?-28 \\
?+15\end{array}$ & $\begin{array}{l}2.0 \\
4.8\end{array}$ & $\begin{array}{l}9.7 \\
7.4\end{array}$ & $\begin{array}{l}7.1 \\
9.4\end{array}$ & $\begin{array}{l}161 \\
413\end{array}$ & $\begin{array}{l}193 \\
522\end{array}$ & $\begin{array}{l}261 \\
739\end{array}$ & $\begin{array}{l}12 \\
39\end{array}$ & $\begin{array}{l}14 \\
49\end{array}$ & $\begin{array}{l}19 \\
70\end{array}$ \\
\hline & M. $\mathbf{3}$. & F & $\begin{array}{l}58 \\
59\end{array}$ & $\begin{array}{l}89.0 \\
93.6\end{array}$ & $\begin{array}{l}2.03 \\
2.06\end{array}$ & $\begin{array}{l}0 \\
180 \mathrm{mg} . \\
14 \mathrm{mos} .\end{array}$ & $\begin{array}{l}-49 \\
+6\end{array}$ & $\underset{5.3}{\ddagger}$ & $\begin{array}{r}10.3 \\
8.9\end{array}$ & $\begin{array}{l}6.7 \\
7.8\end{array}$ & 774 & 650 & 579 & 60 & 50 & 45 \\
\hline & C. ${ }_{4}$. & $\mathbf{M}$ & $\begin{array}{l}74 \\
76\end{array}$ & $\begin{array}{l}69.5 \\
68.9\end{array}$ & $\begin{array}{l}1.79 \\
1.79\end{array}$ & $\begin{array}{l}0 \\
60 \mathrm{mg} . \\
15 \text { mos. }\end{array}$ & -47 & $\begin{array}{l}2.2 \\
2.9\end{array}$ & $\begin{array}{r}12.6 \\
9.4\end{array}$ & $\begin{array}{l}5.5 \\
7.4\end{array}$ & $\begin{array}{l}292 \\
298\end{array}$ & $\begin{array}{l}283 \\
288\end{array}$ & $\begin{array}{l}295 \\
303\end{array}$ & $\begin{array}{l}16 \\
22\end{array}$ & $\begin{array}{l}16 \\
21\end{array}$ & $\begin{array}{l}16 \\
22\end{array}$ \\
\hline & M. $\underset{5}{\mathrm{R}}$. & F & 57 & 74.8 & 1.78 & $45 \mathrm{mg.}$ & -14 & 2.4 & 6.5 & 10.7 & 218 & 212 & 204 & 23 & 22 & 22 \\
\hline & & & 57 & 74.8 & 1.76 & $120 \mathrm{mg}$. & +4 & 4.9 & 5.6 & 13.1 & 458 & 450 & 429 & 57 & 56 & 53 \\
\hline & H. R. & F & $\begin{array}{l}61 \\
62 \\
63\end{array}$ & $\begin{array}{l}63.2 \\
46.4 \\
49.1\end{array}$ & $\begin{array}{l}1.66 \\
1.47 \\
1.50\end{array}$ & $\begin{array}{c}0 \\
0 \\
60 \mathrm{mg} ., \\
15 \text { mos. }\end{array}$ & $\begin{array}{l}-42 \\
-25\end{array}$ & $\begin{array}{l}1.0 \\
2.48 \\
4.28\end{array}$ & $\begin{array}{l}9.3 \\
9.4 \\
8.4\end{array}$ & $\begin{array}{l}7.5 \\
7.4 \\
8.2\end{array}$ & $\begin{array}{l}141 \\
233 \\
379\end{array}$ & $\begin{array}{l}146 \\
274 \\
437\end{array}$ & $\begin{array}{l}157 \\
352 \\
540\end{array}$ & $\begin{array}{l}11 \\
17 \\
31\end{array}$ & $\begin{array}{l}11 \\
20 \\
36\end{array}$ & $\begin{array}{l}12 \\
26 \\
44\end{array}$ \\
\hline & E. W. & F & $\begin{array}{l}74 \\
74\end{array}$ & $\begin{array}{l}68.9 \\
68.0\end{array}$ & $\begin{array}{l}1.73 \\
1.72\end{array}$ & $\begin{array}{l}0 \\
120 \mathrm{mg} . \\
1 \mathrm{mo} .\end{array}$ & $\begin{array}{l}-22 \\
-4\end{array}$ & $\begin{array}{l}2.1 \\
4.8\end{array}$ & $\begin{array}{r}9.3 \\
10.4\end{array}$ & $\begin{array}{l}7.5 \\
6.7\end{array}$ & $\begin{array}{l}228 \\
485\end{array}$ & $\begin{array}{l}228 \\
488\end{array}$ & $\begin{array}{l}232 \\
499\end{array}$ & $\begin{array}{l}17 \\
\mathbf{3 3}\end{array}$ & $\begin{array}{l}17 \\
33\end{array}$ & $\begin{array}{l}17 \\
\mathbf{3 4}\end{array}$ \\
\hline & C. $\mathbf{8}$. & F & 58 & 83.2 & 1.90 & $\begin{array}{l}120 \mathrm{mg} . \\
6 \text { mos. }\end{array}$ & -6 & 3.8 & 6.2 & 11.2 & 392 & 357 & 330 & 44 & 40 & 37 \\
\hline \multirow{12}{*}{ 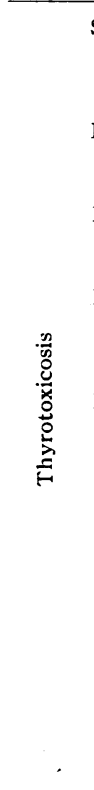 } & S. $\underset{9}{H}$. & F & 44 & 50.8 & 1.50 & 1st I-131, & +10 & 10.18 & 6.3 & 11.0 & 916 & 1,056 & 1,262 & 101 & 116 & \\
\hline & & & 44 & 50.8 & 1.50 & $\begin{array}{l}7 \text { mos. } \\
\text { 2nd I-131, } \\
3 \text { mos. }\end{array}$ & -17 & 4.2 & 9.8 & 7.1 & 253 & 292 & 349 & 18 & 25 & $\begin{array}{r}139 \\
21\end{array}$ \\
\hline & L. D. & F & $\begin{array}{l}17 \\
20\end{array}$ & $\begin{array}{l}52.3 \\
58.0\end{array}$ & $\begin{array}{l}1.52 \\
1.58\end{array}$ & $\begin{array}{l}\text { o } \\
\text { Surg., } \\
1 \text { mo. }\end{array}$ & -19 & $\begin{array}{l}14.6 \\
3.68\end{array}$ & $\begin{array}{l}5.2 \\
6.2\end{array}$ & $\begin{array}{l}13.7 \\
11.2\end{array}$ & $\begin{array}{l}954 \\
266\end{array}$ & $\begin{array}{r}1,088 \\
291\end{array}$ & $\begin{array}{r}1,278 \\
321\end{array}$ & $\begin{array}{r}127 \\
30\end{array}$ & $\begin{array}{r}145 \\
33\end{array}$ & $\begin{array}{r}170 \\
36\end{array}$ \\
\hline & R. D. & F & $\begin{array}{l}31 \\
31\end{array}$ & $\begin{array}{l}51.8 \\
60.8\end{array}$ & $\begin{array}{l}1.54 \\
1.66\end{array}$ & $\begin{array}{l}0 \\
\text { I-131. } \\
12 \text { mos. }\end{array}$ & $\begin{array}{l}+17 \\
-16\end{array}$ & $\begin{array}{r}13.6 \\
1.9\end{array}$ & $\begin{array}{l}4.0 \\
8.8\end{array}$ & $\begin{array}{r}17.3 \\
7.9\end{array}$ & $\begin{array}{l}974 \\
204\end{array}$ & $\begin{array}{r}1,094 \\
213\end{array}$ & $\begin{array}{r}1,315 \\
235\end{array}$ & $\begin{array}{r}169 \\
16\end{array}$ & $\begin{array}{r}190 \\
17\end{array}$ & $\begin{array}{r}228 \\
18\end{array}$ \\
\hline & $\mathrm{M}_{\mathrm{i}} \mathrm{O}$. & $\mathbf{F}$ & 54 & 56.8 & 1.60 & $\begin{array}{l}\text { Drug, } \\
3 \text { weeks }\end{array}$ & +4 & 8.8 & 5.8 & 11.9 & 845 & 913 & 1,039 & 101 & 109 & 124 \\
\hline & & & 55 & 54.5 & 1.58 & $\begin{array}{l}\text { I-131, } \\
15 \text { mos. }\end{array}$ & -16 & 5.1 & 5.4 & 12.8 & 472 & 517 & 606 & 60 & 66 & 77 \\
\hline & L. S. & $F$ & $\begin{array}{l}29 \\
30\end{array}$ & $\begin{array}{l}54.6 \\
74.5\end{array}$ & $\begin{array}{l}1.52 \\
1.78\end{array}$ & $\begin{array}{l}0 \\
\text { I-131, } \\
8 \text { mos. }\end{array}$ & $\begin{array}{l}?+68 \\
?+46\end{array}$ & $\begin{array}{r}11.48 \\
6.98\end{array}$ & $\begin{array}{l}3.7 \\
6.5\end{array}$ & $\begin{array}{l}18.8 \\
10.7\end{array}$ & $\begin{array}{r}1,222 \\
790\end{array}$ & $\begin{array}{r}1,393 \\
768\end{array}$ & $\begin{array}{r}1,564 \\
742\end{array}$ & $\begin{array}{r}229 \\
84\end{array}$ & $\begin{array}{r}261 \\
82\end{array}$ & $\begin{array}{r}293 \\
79\end{array}$ \\
\hline & $\mathrm{M}_{\mathrm{i} 4} \mathrm{~B}$. & $\mathrm{F}$ & $\begin{array}{l}47 \\
49\end{array}$ & $\begin{array}{l}42.3 \\
51.8\end{array}$ & $\begin{array}{l}1.37 \\
1.50\end{array}$ & $\begin{array}{l}\text { 0 } \\
\text { I-131. } \\
14 \text { mos. }\end{array}$ & $\begin{array}{l}+32 \\
-26\end{array}$ & $\begin{array}{c}13.6 \\
6.28\end{array}$ & $\begin{array}{l}4.7 \\
8.2\end{array}$ & $\begin{array}{r}14.7 \\
8.5\end{array}$ & $\begin{array}{l}880 \\
533\end{array}$ & $\begin{array}{r}1,109 \\
615\end{array}$ & $\begin{array}{r}1.461 \\
720\end{array}$ & $\begin{array}{r}129 \\
45\end{array}$ & $\begin{array}{r}163 \\
52\end{array}$ & $\begin{array}{r}214 \\
61\end{array}$ \\
\hline & $\underset{15}{\text { R. M. }}$ & $\mathbf{M}$ & 34 & 61.4 & 1.76 & $\begin{array}{l}\text { I-131, } \\
10 \text { mos. }\end{array}$ & & 4.18 & 9.0 & 7.7 & 350 & 344 & 399 & 27 & 27 & 31 \\
\hline & T. D. & $\mathrm{F}$ & 23 & 72.7 & 1.80 & $\begin{array}{l}\text { I-131. } \\
14 \text { mos. }\end{array}$ & -16 & 4.98 & 6.4 & 10.8 & 726 & 698 & 699 & 78 & 75 & 75 \\
\hline & E. C. & $F$ & 54 & 60.8 & 1.63 & $\begin{array}{l}\text { I-131, } \\
3 \text { mos. }\end{array}$ & -34 & 2.28 & 12.0 & 5.8 & 208 & 221 & 239 & 12 & 13 & 14 \\
\hline & ${ }_{18}$.P. & F & 48 & 63.5 & 1.72 & $\begin{array}{l}\text { I-131, } \\
16 \text { mos. }\end{array}$ & -15 & 4.18 & 9.0 & 7.7 & 262 & 264 & 289 & 20 & 20 & 22 \\
\hline
\end{tabular}

* The patients with myxedema, except M. R., No. 5, had no replacement therapy prior to initial studies (designated "0"') for at least three months. Cases C. L. (No. 4), H. R. (No. 6), and E. W. (No. 7) were previously untreated. H. R. was restudied a second time after omission of therapy for four months. In the studies after treatment, the final dose of desiccated thyroid and the duration on that dose is given without note of preceding lesser doses.

The initial studies on thyrotoxic patients designated " 0 " indicate no therapy other than Lugol's solution. S. H., No. 9, was toxic after one dose of I-131, and M. O. No. 12, was not fully controlled after three weeks of therapy with Tapazole ${ }^{\circledR}$, later propylthiouracil. The periods after I-131 therapy refer to the time after the final dose when more than one was administered.

+ BMR values represent the lowest of two or more determinations in most instances. Discrepant or uncertain determinations are indicated by a question mark.

$\ddagger$ Falsely high PBI, attributed to contamination, prevented calculations from kinetic data.

$\$$ B Itanl extractable iodine (BEI) determinations performed rather than PBI determinations to avoid interference from previously administered inorganic iodine. 


\begin{tabular}{|c|c|c|c|c|c|c|c|c|c|c|c|c|c|c|c|c|}
\hline \multirow[b]{3}{*}{$\underset{\text { tion }}{\text { Condi- }}$} & \multirow[b]{3}{*}{$\begin{array}{c}\text { Sub- } \\
\text { ject }\end{array}$} & \multirow[b]{3}{*}{ Sex } & \multirow[b]{3}{*}{$\begin{array}{l}\text { Age } \\
\text { yrs. }\end{array}$} & \multirow[b]{3}{*}{$\begin{array}{l}\text { Wt. } \\
\text { Kg. }\end{array}$} & \multirow[b]{3}{*}{$\begin{array}{l}\text { Sur- } \\
\text { face } \\
\text { area } \\
M .2\end{array}$} & \multirow[b]{3}{*}{ Therapy* } & \multirow[b]{3}{*}{ BMR† } & \multirow[b]{3}{*}{$\begin{array}{c}\text { PBI } \\
\text { pg. \% }\end{array}$} & \multicolumn{8}{|c|}{ Kinetic and isotope dilution data } \\
\hline & & & & & & & & & \multicolumn{2}{|c|}{$\begin{array}{c}\text { Kinetics of } \\
\text { disappearance }\end{array}$} & \multicolumn{3}{|c|}{$\begin{array}{c}\text { Extrathyroidal organic } \\
\text { iodine pool } \\
\text { (EOI) }\end{array}$} & \multicolumn{3}{|c|}{ Degradation rate } \\
\hline & & & & & & & & & $\begin{array}{c}\text { Half- } \\
\text { time } \\
\text { tz } \\
\text { days }\end{array}$ & $\begin{array}{l}\text { Turn- } \\
\text { over } \\
\text { rate } k \\
\text { \%/day } \\
\end{array}$ & $\mu \mathrm{g}$. & 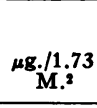 & $\begin{array}{c}\text { Mg./70 } \\
\mathbf{K} \text { g. }\end{array}$ & $\mu \mathrm{g} . / \mathrm{day}$ & $\begin{array}{l}\mu \mathrm{g} . / 1.73 \\
\mathrm{M} .2 / \mathrm{day}\end{array}$ & $\begin{array}{l}\text { Mg./70 } \\
\text { Kg./day }\end{array}$ \\
\hline \multirow{10}{*}{ 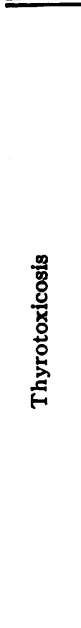 } & P.S. & $\mathbf{M}$ & 38 & 75.5 & 2.00 & $\begin{array}{l}\text { 1-131, } \\
\text { I3 mos. }\end{array}$ & +4 & 6.7 & 6.7 & 10.3 & 929 & 804 & 861 & 96 & 83 & 89 \\
\hline & J. C. & $\mathbf{F}$ & 33 & 53.6 & 1.60 & $\begin{array}{l}\text { Surg., } \\
12 \text { yrs. }\end{array}$ & -15 & 1.98 & 8.3 & 8.3 & 169 & 183 & 221 & 14 & 15 & 18 \\
\hline & $\underset{21}{\text { H. A. }}$ & $\mathbf{M}$ & 48 & 83.6 & 1.94 & $\begin{array}{l}\text { I-131, } \\
6 \text { mos. }\end{array}$ & -15 & 3.6 & 7.3 & 9.5 & 337 & 301 & 282 & 32 & 29 & 27 \\
\hline & A. $Z$. & F & 55 & 71.8 & 1.64 & $\begin{array}{l}\text { I-131, } \\
19 \text { mos. }\end{array}$ & -7 & 5.5 & 7.6 & 9.1 & 429 & 453 & 418 & 39 & 41 & 38 \\
\hline & R. R. & F & 39 & 57.3 & 1.66 & $\begin{array}{l}\text { I-131, } \\
4 \text { mos. }\end{array}$ & +4 & $4.8 \S$ & 7.8 & 8.9 & 418 & 436 & 511 & 37 & 39 & 45 \\
\hline & O. 24 & F & 43 & 72.7 & 1.90 & $\begin{array}{l}\text { I-131, } \\
14 \text { mos. }\end{array}$ & -16 & 5.28 & 6.9 & 10.0 & 535 & 487 & 515 & 54 & 49 & 52 \\
\hline & D. B. & $\mathrm{F}$ & 48 & 53.2 & 1.46 & $\begin{array}{l}\text { Surg., } \\
2 \text { mos. }\end{array}$ & $?+25$ & 6.48 & 7.0 & 9.9 & 649 & 769 & 854 & 64 & 76 & 84 \\
\hline & A. V. & $\mathbf{M}$ & 59 & 72.7 & 1.80 & $\begin{array}{l}\text { I-131, } \\
13 \text { mos. }\end{array}$ & +12 & 6.18 & 7.0 & 9.9 & 557 & 535 & 536 & 55 & 53 & 53 \\
\hline & P. L. & F & 51 & 50.9 & 1.51 & $\begin{array}{l}\text { I-131; } \\
7 \text { mos. }\end{array}$ & +5 & 5.58 & 7.0 & 9.9 & 412 & 472 & 567 & 41 & 47 & 56 \\
\hline & L. T. & $\mathbf{M}$ & 55 & 75.7 & 1.85 & $\begin{array}{l}\text { I-131, } \\
5 \text { mos. }\end{array}$ & -8 & 5.3 & 7.6 & 9.1 & 647 & 605 & 598 & 59 & 55 & 55 \\
\hline 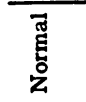 & \multicolumn{8}{|c|}{$\begin{array}{l}\text { Mean of } 10 \text { subjects } \\
\text { Standard deviation } \\
\text { Standard error of mean }\end{array}$} & $\begin{array}{l}6.6 \\
0.7 \\
0.2\end{array}$ & $\begin{array}{r}10.7 \\
1.1 \\
0.4\end{array}$ & $\begin{array}{r}557 \\
99 \\
31\end{array}$ & $\begin{array}{r}501 \\
84 \\
27\end{array}$ & $\begin{array}{r}521 \\
87 \\
28\end{array}$ & $\begin{array}{l}59 \\
11 \\
3.3\end{array}$ & $\begin{array}{c}53 \\
9 \\
2.9\end{array}$ & $\begin{array}{c}55 \\
9 \\
2.9\end{array}$ \\
\hline
\end{tabular}

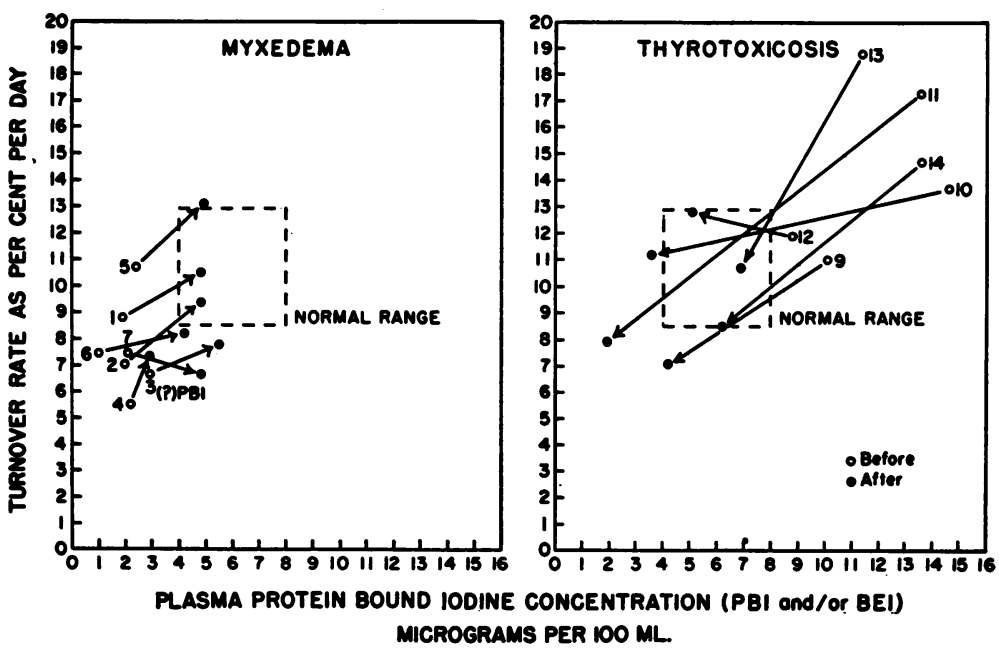

Fig. 5. Relation Between Thyroxine Turnover Rate and PBi Before and After Treatment

The normal PBI range was taken as 4 to 8 micrograms per $100 \mathrm{ml}$. The normal range of turnover rates (8.5 to 12.9 per cent per day) was obtained from the normal mean $(10.7 \pm 1.1$ per cent per day), plus and minus two standard deviations. The rate of fractional turnover was selected as the ordinate since this is a purely kinetic parameter computed independently of the PBI value. It will be noted that all changes in each group are in the same direction except No. 7 (E.W.) in the myxedema group, and No. 12 (M.O.) in the thyrotoxic group. 
This was also the case in subject P.S., No. 19 (Table I), who had a normal turnover rate but large pool size and degradation rate. With the exceptions of M.O. (No. 12), L.S. (No. 13) and P.S. (No. 19), the remaining 17 subjects all showed reductions in turnover rate, pool size and degradation rate either to the normal range or below it.

Figure 3 was selected to illustrate an instance of a very slow turnover following treatment. Subject R.D., No. 11, was clinically hypothyroid, and the PBI, turnover kinetics, pool size and degradation rate were all within the range seen in spontaneously occurring myxedema (Table I). This was also true of Subject E.C., No. 17, who appeared clinically hypothyroid, and Subject J.C., No. 20, who appeared euthyroid 12 years after thyroidectomy, despite a low PBI value. Subjects R.M. (No. 15), E.P. (No. 18) and S.H. (No. 9) also had low kinetics, pool sizes and degradation rates but with normal PBI values and euthyroid clinical appearances.

The time interval after treatment varied. Subject L.D., No. 10, was restudied only one month after subtotal thyroidectomy and revealed marked alterations, illustrating the rapidity with which such changes could occur.

\section{Myxedema}

In contrast, the changes were slower to appear in the myxedematous patients on replacement therapy. In general the curves and values were found to have reached or approached the normal range only after considerable periods on replacement therapy. Thus, Subject H.L., No. 1 (Figure 4 and Table I), showed change to essentially normal kinetic, pool and degradation values after therapy for a year. Subject M.A., No. 2, likewise reverted to normal values after a year's treatment which consisted of $90 \mathrm{mg}$. desiccated thyroid daily during the final six months. In contrast, Subject E. W., No. 7 (Table I), showed an even slower disappearance rate when restudied after four months on desiccated thyroid (one month on $120 \mathrm{mg}$. daily); although the PBI and pool size had reached normal, the degradation rate was still low.

Subject M.R., No. 5, was studied initially on incomplete replacement therapy. When restudied after five months on increasing doses of desic- cated thyroid (one month on $120 \mathrm{mg}$. daily) there was acceleration of turnover and increase to normal pool and degradation values.

Subject C.L., No. 4, was maintained at a hypothyroid level because of a myocardial infarction. After 15 months of desiccated thyroid $(60 \mathrm{mg}$. daily) there was a definite acceleration of turnover, although still in the hypothyroid range. The pool size was unchanged but the degradation was increased due to the accelerated turnover.

Subject C.S., No. 8, showed all values within the normal range. Subject M.M., No. 3, had a low turnover but normal degradation rate by virtue of a high normal pool.

\section{DISCUSSION}

In the previous study (1) the metabolism of $\mathrm{I}^{131}$-labeled 1-thyroxine was strikingly different from normal in the hyper- and hypothyroid states. These differences were evident in the turnover rates as per cent per day, the pools of extrathyroidal hormone, and the degradation rates which represented products of pools and turnovers. All parameters were higher than normal in thyrotoxicosis, and lower in myxedema.

The present study showed quite marked changes after treatment, especially in the thyrotoxic subjects. Reversion to the normal range was seen as early as one month after subtotal thyroidectomy. Nineteen of the 20 thyrotoxic subjects had posttherapy values approximating the normal range or below it, in three instances duplicating the findings seen in spontaneous myxedema.

The exceptional patient M.O., No. 12 (Figure 2 ), showed a slightly accelerated turnover rate both in an initial study while maintained nearly euthyroid on drugs, and in the final study. Although the disappearance curves were essentially the same, the pool size and degradation rate became much smaller. Determination of thyroxinebinding protein (TBP) through the courtesy of Dr. Jacob Robbins revealed no abnormality in this patient's serum. With the exception of this one instance, the present findings of marked changes after therapy of thyrotoxicosis did not agree with the preliminary report of Ingbar and Freinkel (6) who observed accelerated turnovers in most patients after therapy.

Although myxedematous subjects on replacement therapy did reveal changes to or toward 
the normal range, these changes occurred more slowly than with treatment of thyrotoxicosis. The previous report (1) demonstrated that rapid massive replacement therapy with triiodothyronine and thyroxine failed to alter disappearance curves in studies continued 7 to 10 days after treatment. Thus, there was no change in fractional turnover rate despite increased absolute amount of hormone removed with elevation of PBI concentration. Subsequent similar studies ( 7 ) continued as long as one month showed no change in plasma disappearance curves, nor in urinary excretion of the radioactive label. Moreover, these findings have been confirmed and extended by Richards, Freinkel and Ingbar (8). It is therefore quite evident that the turnover curves of myxedematous subjects are changed only by relatively prolonged replacement therapy.

In the previous report (1) it was suggested that peripheral metabolism, or some function thereof, had importance in determining hormone degradation rate. This was considered plausible in view of elevated degradation rates observed in hypermetabolic states such as acute leukemia with normal PBI concentration. Such a concept also received support from the observation by Castor and Beierwaltes (9) that dinitrophenol administration for two days, producing marked hypermetabolism, was associated with a fall in PBI concentration in normal human subjects and in an athyreotic subject maintained euthyroid on replacement therapy.

A possible mechanism is suggested by the work of Larson, Tomita and Albright (10) in which kidney slices from thyroidectomized rats converted thyroxine to triiodothyronine more slowly than slices from normal rats, while the kidneys from thyrotoxic animals (fed desiccated thyroid or exposed to cold) accomplished this conversion at an accelerated rate. The postulated adaptive enzyme system for thyroxine deiodination may well have relevance for human disappearance curve studies.

An alternative hypothesis to account for differences in hormone degradation rate pertains to the concentration of the thyroxine binding protein of plasma. The possible role of the thyroxine binding protein (TBP) was previously mentioned (1) and TBP has received increasing attention in recent work. The findings of Hamol- sky, Ellison and Freedberg with regard to uptake of labeled triiodothyronine from plasma by erythrocytes (11) or diaphragm (12) may reflect variations in thyroxine bound to TBP. The studies of Albright, Larson and Deiss (13) have not suggested that binding capacity differs from normal in the hypo- or hyperthyroid state. On the other hand, Robbins and Rall, using reverse-flow electrophoresis, found the absolute TBP concentration slightly higher than normal in myxedema, but not different from normal in hyperthyroidism (14).

In any event, where plasma thyroxine concentration is higher, one might expect that the extent of "saturation" of TBP would be greater. The thyroxine not bound to TBP is "non-specifically" bound to albumin. The existence of a minute amount of "unbound" or "free" thyroxine has been postulated by Robbins and Rall based upon computations of protein association constants. Moreover, these authors have suggested that the amount of "free" thyroxine in the plasma may determine the thyroxine degradation rate, and have presented calculations compatible with such a hypothesis. On the other hand, the failure of very large doses of intravenous thyroxine to alter the turnover rate, as noted above (1), would appear to argue against such a conception. The attainment of PBI values as high as $26 \mathrm{mi}$ crograms per cent (1) would be expected to provide much "free" thyroxine despite possible increased thyroxine binding capacity of myxedematous plasma (14).

The data and inferences of the author, cited above, receive support from the work by Ingbar's group (8) which includes observations of displacement of thyroxine from TBP onto albumin after thyroxine loading. Despite abrupt increase of the thyroxine pool after loading, the fractional rate of turnover was unaffected. In contrast, subjects made hypermetabolic by prolonged administration of triiodothyronine showed accelerated thyroxine turnover despite reduction in PBI and computed "free" thyroxine values.

Such observations suggest that factors other than TBP must be implicated in thyroxine turnover. Recent work has underscored the need for further clarification of the nature of the binding protein or proteins. Employing starch gel electrophoresis, Rich and Bearn (15) have reported 
that the first prealbumin band contained 60 per cent of the radioactivity of added radiothyroxine, and had a binding capacity far in excess of that previously reported for the interalpha zone. Ingbar (16) used a different electrophoretic method to reveal thyroxine bound in both interalpha and prealbumin areas, the latter possessing greater binding capacity, which, however, was diminished in untreated Graves' disease.

Future work will be required to settle definitely whether plasma protein binding or some ratelimiting step related to peripheral tissue metabolism is of paramount importance in determining thyroxine turnover.

A further hypothesis, embracing both of these alternatives, would assign a significant role to the turnover rate of the thyroxine binding protein itself. This would be consistent with previous evidence on serum protein metabolism, such as $\mathrm{I}^{131}$-tagged albumin turnover studies which have demonstrated the accelerating effect of thyroid hormone $(7,17,18)$.

\section{SUMMARY}

1. The disappearance curve of plasma radioactivity after intravenous injection of $\mathrm{I}^{131}$-labeled 1-thyroxine was employed to determine the rate of hormone degradation or removal.

2. Thyroxine degradation was slower than normal in untreated myxedema, and faster than normal in thyrotoxicosis.

3. In 19 of 20 cases of thyrotoxicosis treated with radioiodine or surgery, the degradation rate became approximately normal or even below normal. Pronounced changes were observed as rapidly as one month after subtotal thyroidectomy. In a single instance, the disappearance curve remained slightly accelerated after attainment of euthyroidism.

4. In two cases of postradioiodine hypothyroidism and one post thyroidectomy patient, the rate was as slow as that seen in spontaneously occurring myxedema.

5. In myxedematous patients on replacement therapy, the disappearance curve might remain unaltered for as long as one to four months despite attainment of euthyroid status. After prolonged replacement therapy of myxedema for six months or more, the degradation rates became normal or changed in the direction of normal.

6 . The results suggested that the rate of removal of circulating thyroxine is a significant parameter of hormone metabolism possibly reflecting a function of peripheral tissue metabolism and/or extent of association with thyroxine-binding protein.

\section{ACKNOWLEDGMENTS}

The author wishes to express gratitude for the valuable technical assistance of Mr. Bruce Wright, Mr. Joseph Martino, and Mrs. Sondra Rose Kluger.

\section{REFERENCES}

1. Sterling, K., and Chodos, R. B. Radiothyroxine turnover studies in myxedema, thyrotoxicosis, and hypermetabolism without endocrine disease. J. clin. Invest. 1956, 35, 806.

2. Berson, S. A., and Yalow, R. S. Quantitative aspects of iodine metabolism. The exchangeable organic iodine pool, and the rates of thyroidal secretion, peripheral degradation and fecal excretion of endogenously synthesized organically bound iodine. J. clin. Invest. 1954, 33, 1533.

3. Ingbar, S. H., and Freinkel, N. Simultaneous estimation of rates of thyroxine degradation and thyroid hormone synthesis. J. clin. Invest. 1955, 34, 808.

4. Barker, S. B., Humphrey, M. J., and Soley, M. H. The clinical determination of protein-bound iodine. J. clin. Invest. 1951, 30, 55.

5. Man, E. B., Kydd, D. M., and Peters, J. P. Butanolextractable iodine of serum. J. clin. Invest. 1951, $30,531$.

6. Ingbar, S. H., and Freinkel, N. An abnormality of the peripheral metabolism of thyroxine in patients with treated Graves' disease: The syndrome of euthyroidism associated with thyroidal hyperfunction (abstract). J. clin. Invest. 1955, 34, 914.

7. Sterling, K. Unpublished work.

8. Richards, J. B., Freinkel, N., and Ingbar, S. H. The effect of thyroxine loading on protein-binding and peripheral turnover of thyroxine in man (abstract). Clin. Res. 1958, 6, 243.

9. Castor, C. W., and Beierwaltes, W. H. Effect of dinitrophenol on thyroid function in man. J. clin. Endocr. 1956, 16, 1026.

10. Larson, F. C., Tomita, K., and Albright, E. C. The deiodination of thyroxine to triiodothyronine by kidney slices of rats with varying thyroid function. Endocrinology 1955, 57, 338.

11. Hamolsky, M. W., Stein, M., and Freedberg, A. S. The thyroid hormone-plasma protein complex in man. II. A new in vitro method for study of "uptake" of labelled hormonal components by human erythrocytes. J. clin. Endocr. 1957, 17, 33. 
12. Hamolsky, M. W., Ellison, H. E., and Freedberg, A. S. The thyroid hormone-plasma protein complex in man. I. Differences in different states of thyroid function. J. clin. Invest. 1957, 36, 1486.

13. Albright, E. C., Larson, F. C., and Deiss, W. P. Thyroxine binding capacity of serum alpha globulin in hypothyroid, euthyroid, and hyperthyroid subjects. J. clin. Invest. 1955, 34, 44.

14. Robbins, J., and Rall, J. E. The interaction of thyroid hormones and protein in biological fluids. Recent Prog. Hormone Res. 1957, 13, 161.

15. Rich, C., and Bearn, A. G. Localization of the thyroxine-binding protein of serum by starch gel electrophoresis. Endocrinology 1958, 62, 687.
16. Ingbar, S. H. Pre-albumin, a new thyroxine-binding protein of human plasma: Its isolation and physiologic activity in normal and abnormal states (abstract). J. clin. Invest. 1958, 37, 903.

17. Schwartz, E. The effect of thyroid hormone upon the degradation rate and miscible pool of radioiodinated human serum albumin in myxedema. J. Lab. clin. Med. 1955, 45, 340.

18. Rothschild, M. A., Bauman, A., Yalow, R. S., and Berson, S. A. The effect of large doses of desiccated thyroid on the distribution and metabolism of albumin- $\mathrm{I}^{121}$ in euthyroid subjects. J. clin. Invest. 1957, 36, 422.

\section{CORRECTION}

In the paper by Gold, Singleton, Macfarlane and Moore entitled "Quantitative Determination of the Urinary Cortisol Metabolites, 'Tetrahydro F,' 'Allo-Tetrahydro F' and 'Tetrahydro E': Effects of Adrenocorticotropin and Complex Trauma in the Human" in Volume 37, Pages 813 and 822. The chemical name for cortisol should be $11 \beta, 17 \alpha, 21$ trihydroxy $\Delta^{4}$ pregnene-3,20-dione, rather than $11 \beta, 17 \alpha, 21$ trihydroxy-pregnane-3,20 dione ; and that for cortisone should be $17 \alpha, 21$ dihydroxy $\Delta^{4}$ pregnene-3,11,20-trione, rather than $17 \alpha, 21$ dihydroxy-pregnane-3,11,20 trione. 